\title{
Introduction to the sense of taste in endocrinology
}

\author{
Christian A. Koch ${ }^{1,2}$
}

Published online: 30 August 2016

(C) Springer Science+Business Media New York 2016

Increasing rates of obesity worldwide are alarming and are among the driving forces for increasing health care costs, especially in developed countries. It is puzzling that the number of people with a body mass index above 30 has further increased despite the increasing use of artificial sweeteners over the last several decades. The sense of taste and hedonics are important in this setting and it is with great honor to introduce guest editors and expert consultants Dr. Kristina Rother and Dr. Valerie Duffy who have both recruited an outstanding group of contributing authors for this special issue, demonstrating the intersection between endocrinology and various other fields of the biological sciences.

Drs. Snyder and Bartoshuk, both from the Institute of Food and Agricultural Sciences at the University of Florida, Gainesville, have provided an outstanding overview of oral sensory function. The impact of otitis media, head trauma, vascular accidents such as stroke, and of other conditions on the taste system is emphasized.

Next, the existence of a sixth taste modality, a taste of fat, and its relationship to obesity is reviewed by Dr. Besnard from the INSERM and NUTox in France.

Dr. Julie Mennella and colleagues from the Monell Chemical Senses Center in Philadelphia provide an excellent article on the development of sweet taste and its power to blunt expressions of pain and to mask bad tastes in foods, helping us understand childrens unique vulnerability to our

Christian A. Koch

ckoch@umc.edu

1 Department of Medicine, Division of Endocrinology, Diabetes, and Metabolism, University of Mississippi Medical Center, 2500 N State Street, Jackson, MS 39216, USA

2 G.V. (Sonny) Montgomery VA Medical Center, Jackson, MS 39216 , USA current food environment. This leads us to the question whether and how artificial sweeteners cause metabolic dysregulation which Drs. Shearer (University of Calgary, Canada) and Swithers (Purdue University, Indiana) reviewed with an emphasis on mechanistic data from animal models and including agricultural settings and human studies.

Acknowledging that well-controlled intervention studies that rigorously evaluate the health effects of low calorie sweeteners in humans are limited, Dr. Rother and colleagues from the National Institutes of Health provide an overview on methodological considerations in this setting with a focus on interventional studies of low calorie sweetener effects on metabolism and weight.

Considering that lifestyle modifications regarding energy balance often fail longterm for overweight and obese individuals and that metabolic surgery is being increasingly utilized, the question arises whether taste and food preferences are altered following bariatric surgery. This is reviewed by Dr. Hsia and colleagues from the Pennington Biomedical Research Center in Baton Rouge, LA, and the Louisiana State University Health Sciences Center in New Orleans.

Dr. Pirastu from Trieste, Italy, and his colleagues from the Netherlands and the San Raffaele Scientific Institute in Milan, Italy, assessed 20 specific food likings in one of the first large scale genome wide association studies on food likings, utilizing a two step metaanalysis including populations from Italy, the Netherlands, and Central Asia.

The concluding article represents an overview of the U.S. National Health and Nutrition Examination Survey (NHANES) regarding chemosensory assessments and olfactory exam findings in people older than age 40 years, authored by guest editor Dr. Duffy from the Department of Allied Health Sciences in Storrs, CT, and colleagues from the National Institutes of Health. 
Professor Christian A. Koch

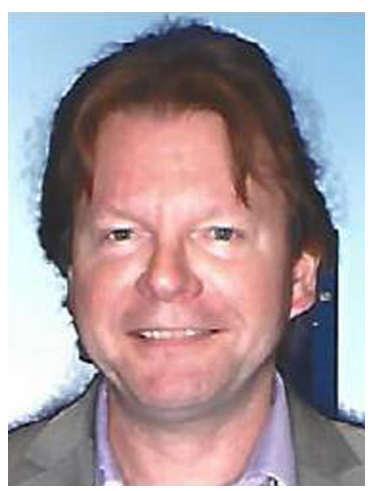

Prof. Koch's research and clinical interest areas include endocrine hypertension including metabolic syndrome and obesity and secondary and atypical forms of diabetes mellitus. Prof. Koch trained in neurosurgery, neurology, and internal medicine in Germany, and completed a residency in Internal Medicine at the Ohio State University in Columbus, OH, USA, under the chairmanship of Prof. EL Mazzaferri, and clinical and research training in endocrinology at the National Institutes of Health in Bethesda, MD, under the guidance of Prof. George P. Chrousos. In 2002, Koch returned to Germany and joined the division of Prof. Ralf Paschke (since 2004 Prof. M. Stumvoll) as C2 faculty Oberarzt at the University of Leipzig and then Adjunct Associate Professor at Georgetown University, before taking on the position as director of the division of endocrinology at the University of Mississippi in Jackson, MS, in 2006, a position he has held until 2013, when he obtained tenure. Prof. Koch's focus remains translational and multidisciplinary research with questions arising from patient encounters, trying to explain clinical observations by looking at "bench" results to further improve patient care.

Dr. Kristina I. Rother

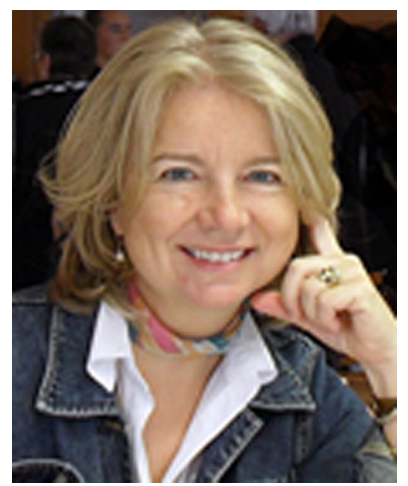

Website:

https://www.niddk.nih.gov/about-niddk/staff-directory/ intramural/kristina-rother/Pages/research-summary.aspx

Dr. Rother has graduated from Medical School at the University of Freiburg, Germany, in 1986 and completed her medical dissertation ( $\mathrm{PhD}$ equivalent) in the same year before starting a residency in pediatrics at the Mayo Clinic, Rochester, MN. She completed a fellowship in pediatric endocrinology at the Massachusetts General Hospital, the Mayo Clinic, and the National Institutes of Health. In 2000, she became Clinical Investigator at the NIH and is Chief of the Section on Pediatric Diabetes \& Metabolism. In 2008, she obtained the degree Master of Health Sciences in Clinical Research at the NIH and Duke University. Dr. Rother's research focus comprises pathophysiologic mechanisms and therapeutic interventions for type 1 and type 2 diabetes as well as obesity. Additional topics of interest include the endocrine features of rare diseases associated with lipodystrophy, e.g., PIK3CA-related overgrowth disorders, dermatomyositis and CANDLE syndrome. One of Dr. Rother's current main focus areas is the elucidation of health effects of artificial sweeteners.

Professor Valerie Duffy, PhD, RD

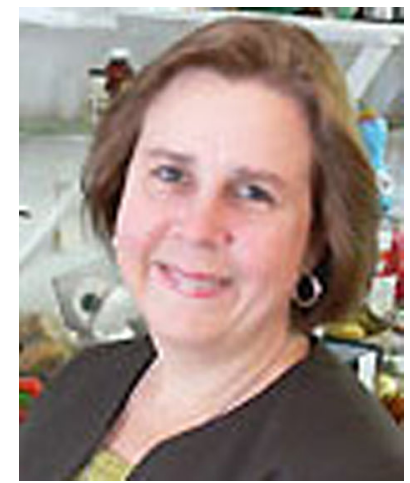

Website:

http://alliedhealth.uconn.edu/facultyAndStaff/ ValerieDuffy.php

Prof. Valerie B. Duffy (PhD, RD) is Director of the Graduate Program in the Allied Health Sciences jointly appointed in Nutritional Sciences and offers a wealth of experience in food, nutrition, health promotion and public health nutrition. Prof. Duffy trained at Cornell University, New York, Rush-Presbyterian-St. Luke's Medical Center in Chicago, and obtained her PhD (human nutrition) at the University of Connecticut in 1992. She also worked as a postdoc on the topics taste psychophysics and taste neurobiology at Yale University. The Duffy Lab at the University of Connecticut has two main research interests. First, attempting to understand variation in chemosensory perception in humans and how this variation influences food flavor, food preference and food intake. More recently, studying how chemosensory variation influences responses to flavored cigarettes and e-cigarettes as well as bariatric surgery. The ultimate goal of the Duffy lab is to understand how chemosensory variation influences the ability to 
follow a healthy diet and behaviors for the prevention of chronic disease and obesity. Prof. Duffy and her team also collaborate with community agencies across the state to promote healthy diets and healthy weights of children, particularly those of economic disadvantage.
Through involvement of undergraduate and graduate student research, the Duffy team are investigating the effectiveness of community-based interventions to improve vegetable intake and level of physical activity of children. 